Article

\title{
Performance of the IOTA ADNEX Model on Selected Group of Patients with Borderline Ovarian Tumours
}

\author{
Adrius Gaurilcikas ${ }^{1, *}$, Migle Gedgaudaite ${ }^{1}$, Arvydas Cizauskas ${ }^{2}$, Vaida Atstupenaite ${ }^{3}$, \\ Saulius Paskauskas ${ }^{1}$, Dovile Gaurilcikiene ${ }^{1}$, Tomas Birzietis ${ }^{1}$, Daiva Vaitkiene ${ }^{1}$ (D) \\ and Ruta Jolanta Nadisauskiene ${ }^{1}$ \\ 1 Department of Obstetrics and Gynaecology, Medical Academy, Lithuanian University of Health Sciences, \\ LT-44307 Kaunas, Lithuania; migle.gedgaudaite@lsmuni.lt (M.G.); saulius.paskauskas@lsmu.lt (S.P.); \\ divid.d@gmail.com (D.G.); tomas.birzietis@lsmu.lt (T.B.); daiva.vaitkiene@lsmuni.lt (D.V.); \\ ruta.nadisauskiene@lsmuni.lt (R.J.N.) \\ 2 Department of Pathological Anatomy, Medical Academy, Lithuanian University of Health Sciences, \\ LT-44307 Kaunas, Lithuania; arvydas.cizauskas@1smu.lt \\ 3 Department of Radiology, Medical Academy, Lithuanian University of Health Sciences, \\ LT-44307 Kaunas, Lithuania; vaida.atstupenaite@lsmu.lt \\ * Correspondence: adrius.gaurilcikas@lsmu.lt; Tel.: +37-06-398-9530
}

Received: 27 October 2020; Accepted: 3 December 2020; Published: 11 December 2020

\begin{abstract}
Background and objectives: ultrasound is considered to be the primary tool for preoperative assessment of ovarian masses; however, the discrimination of borderline ovarian tumours (BOTs) is challenging, and depends highly on the experience of the sonographer. The Assessment of Different NEoplasias in the adneXa (ADNEX) model is considered to be a valuable diagnostic tool for preoperative assessment of ovarian masses; however, its performance for BOTs has not been widely studied, due to the low prevalence of these tumours. The aim of this study was to evaluate the performance of ADNEX model for preoperative diagnosis of BOTs. Methods: retrospective analysis of preoperative ultrasound datasets of patients diagnosed with BOTs on the final histology after performed surgery was done at a tertiary oncogynaecology centre during the period of 2012-2018. Results: 85 patients were included in the study. The performance of ADNEX model based on absolute risk (AR) improved with the selection of a more inclusive cut-off value, varying from $47(60.3 \%)$ correctly classified cases of BOTs, with the selected cut-off of $20 \%$, up to $67(85.9 \%)$ correctly classified cases of BOTs with the cut-off value of $3 \%$. When relative risk (RR) was used to classify the tumours, $59(75.6 \%)$ cases were identified correctly. Forty $(70.2 \%)$ cases of serous and $16(72.7 \%)$ cases of mucinous BOTs were identified when AR with a $10 \%$ cut-off value was applied, compared to 44 $(77.2 \%)$ and $15(68.2 \%)$ cases of serous and mucinous BOTs, correctly classified by RR. The addition of Ca125 improved the performance of ADNEX model for all BOTs in general, and for different subtypes of BOTs. However, the differences were insignificant. Conclusions: The International Ovarian Tumour Analysis (IOTA) ADNEX model performs well in discriminating BOTs from other ovarian tumours irrespective of the subtype. The calculation based on RR or AR with the cut-off value of at least 10\% should be used when evaluating for BOTs.
\end{abstract}

Keywords: borderline ovarian tumours; ultrasound; transvaginal ultrasound; preoperative assessment; ADNEX

\section{Introduction}

Compared to invasive ovarian cancer, borderline ovarian tumours (BOTs) are associated with a significantly better overall survival rate (59.7-99.6\%, depending on the stage of the disease) [1,2], but tend to compromise younger patients [1-4]. The possibility of conservative management and fertility 
preservation makes preoperative diagnosis of BOTs a very important obstacle [5,6]. Ultrasound is considered to be the primary tool for preoperative assessment of ovarian masses $[7,8]$; however, the discrimination of BOTs is challenging, and the accuracy of the diagnosis depends highly on the experience of the sonographer [7,9].

In 2014, the International Ovarian Tumour Analysis (IOTA) group delivered the Assessment of Different NEoplasias in the adneXa (ADNEX) model, which is the first risk model to differentiate benign ovarian tumours, BOTs, stage I invasive cancer, stage II-IV invasive ovarian cancer, and secondary metastatic cancer [10].

For the calculation of risk, the ADNEX model uses three clinical variables (patients' age, Ca125 tumour marker, and whether the ultrasound scan was performed in an oncology center or not) and six ultrasound variables (maximal diameter of the lesion, maximal diameter of the largest solid part, presence of more than 10 locules (Figure 1) in the ovarian lesion, number of papillary projections (Figure 2), presence of acoustic shadows, and ascites) [10].

The ADNEX model is considered a valuable diagnostic tool for preoperative assessment of ovarian masses, especially in the clinical setting with less experienced sonographers. However, its performance for BOTs has still not been widely studied, due to the low prevalence of these tumours.

The aim of this study was to evaluate the performance of ADNEX model for preoperative diagnosis of BOTs.

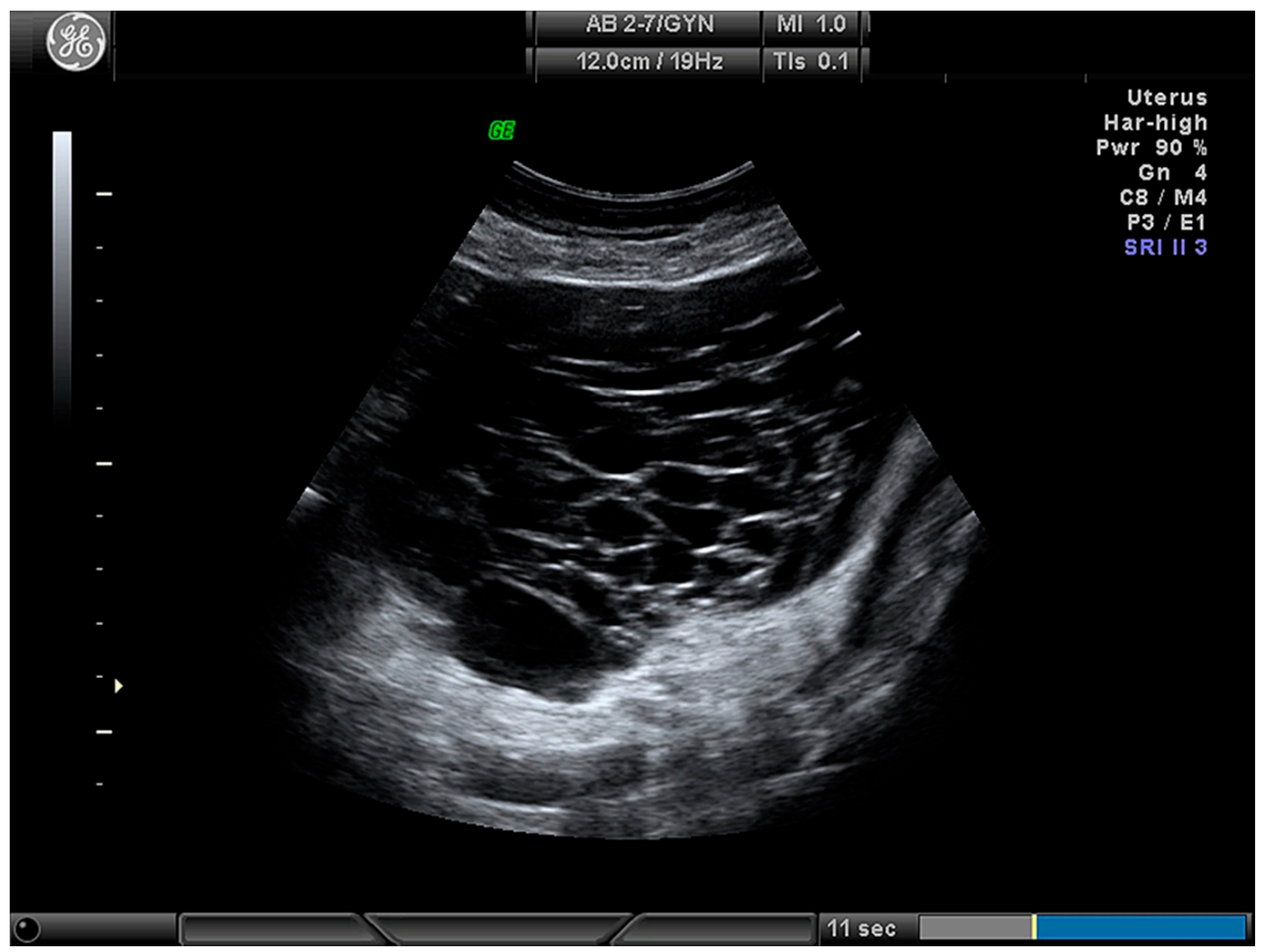

Figure 1. Multilocular ovarian lesion with more than 10 locules. 


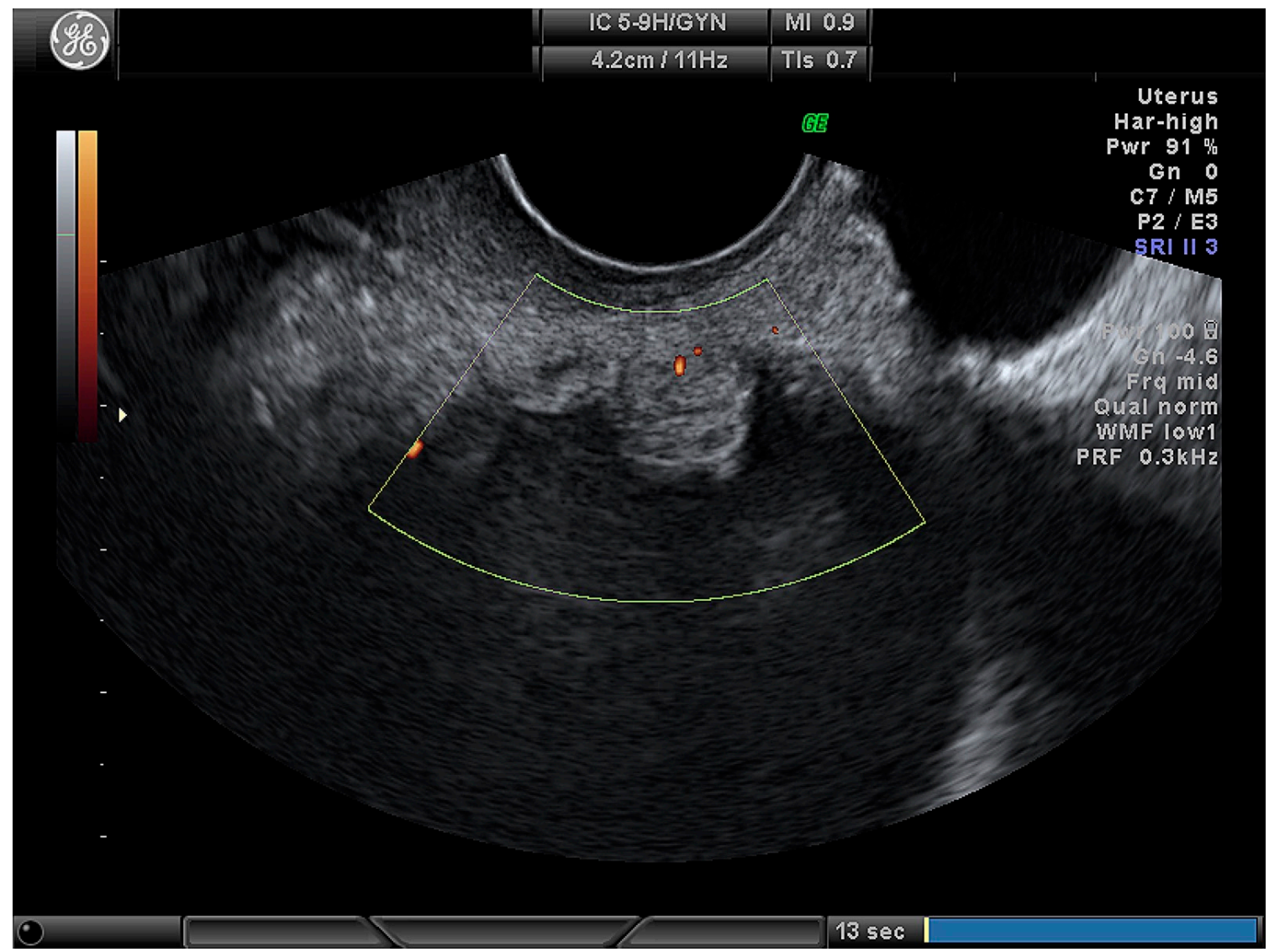

Figure 2. Papillary projection in ovarian lesion.

\section{Materials and Methods}

\subsection{Ethics}

The study was conducted in accordance with the Declaration of Helsinki, and the protocol was approved by the Bioethics Centre of Lithuanian University of Health Sciences (BEC-MF-88).

\subsection{Design}

A retrospective analysis was conducted at Lithuanian University of Health Sciences, Kaunas, Lithuania.

\subsection{Patients and Ultrasound Collection Parameters}

Ultrasound (US) datasets of patients diagnosed with a BOT on the final histology after performed surgery was done, during the period of 2012-2018, were used. Only the patients with primary BOTs were included into the study. The following US parameters were collected: largest diameter of the mass, largest diameter of the solid part of the mass, number of papillary projections, number of locules, presence of ascites, and acoustic shadows.

\subsection{IOTA ADNEX Model Specifications and Interpretation}

The IOTA ADNEX model (web application) was used to calculate the absolute risk (AR), predicting the probability of the mass being a BOT. To evaluate the performance of ADNEX model by AR, different cut-off values $(20 \%, 10 \%, 5 \%$, and $3 \%)$ for the risk of malignancy were tested. The test result was evaluated as "positive" if the risk for BOT was the highest among malignant ovarian tumour groups for each selected cut-off value. The relative risk (RR) was calculated as a proportion of absolute 
risk and population risk. According to RR, the test was evaluated as "positive" if the risk for a BOT was the highest among benign, invasive, and metastatic ovarian tumour groups. Additionally, the performance of the ADNEX model was evaluated with and without a preoperative level of Ca125 tumour markers (selected cut-off value for malignancy for AR was 10\%). Seven cases with missing Ca125 levels were excluded from the calculations.

To evaluate the performance of the ADNEX model in different types of BOTs, the study group was divided into two subgroups, according to the final histology: serous BOTs and mucinous BOTs. Due to the low number, mixed and endometrioid BOTs were excluded from later calculations $(n=6)$. The ARs (10\% cut-off value for malignancy has been selected) and RRs, with and without added preoperative levels of Ca125, were calculated for the subgroups of serous and mucinous BOTs, as described previously.

\subsection{Statistical Analysis}

All calculations were made using Statistical Package of Social Sciences, Mac version 26 (SPSS, IBM, Brøndby, Denmark). Continuous variables were described using the median (interval), and categorical variables were reported as frequency and percentage. As data were not distributed normally, a Mann-Whitney U test was used for pairwise comparisons. Pearson's Chi-square test was used to compare independent categorical variables, while Cochrane's $Q$ test and McNemar criteria were used to compare the related ones. The statistical significance level was a $p$ value less than 0.05 .

\section{Results}

\subsection{Main Characteristics of Study Population}

Eighty-five patients were included in the study. The main characteristics of the patients are shown in Table 1.

Table 1. Clinical characteristics of the study population.

\begin{tabular}{cc}
\hline Median Age (Range) & $\mathbf{4 6 . 1}(\mathbf{1 7 - 8 3 )}$ \\
\hline Tumours histology: \\
Serous \\
Mucinous & $57(67.1 \%)$ \\
Other: & $22(25.9 \%)$ \\
Endometrioid & $4(4.7 \%)$ \\
Mixed & $2(2.3 \%)$ \\
\hline FIGO Stage: & $74(87.1 \%)$ \\
I & $11(12.9 \%)$ \\
\hline II-III & $72.8(5.9-918.0){ }^{*}$ \\
\hline * Seven cases were missing a Ca125 value, and were excluded from the analysis when Ca125 was needed.
\end{tabular}

The main ultrasound features of the BOTs are presented in Tables 2 and 3.

Table 2. Ultrasound features of borderline ovarian tumours (BOTs).

\begin{tabular}{cc}
\hline Ultrasound Feature & Median (Interval) or $\boldsymbol{n} \mathbf{( \% )}$ \\
\hline Maximal diameter of the tumours $(\mathrm{mm})$ & $80.0(20-639)$ \\
\hline Maximal diameter of the solid part $(\mathrm{mm})$ & $15.0(3-73)$ \\
\hline Type of tumour: & $24(28.2)$ \\
Cystic (Figure 3) & $61(71.8)$ \\
\hline Cystic-solid (Figure 4) &
\end{tabular}


Table 2. Cont

\begin{tabular}{cc}
\hline Ultrasound Feature & Median (Interval) or $\boldsymbol{n} \mathbf{( \% )}$ \\
\hline Number of locules: & \\
Unilocular & $41(48.2)$ \\
Multiloculcar (total) & $44(51.8)$ \\
More than 10 locules & $30(35.3)$ \\
\hline Number of papillary projections: & \\
None & $30(35.3)$ \\
1 & $15(17.6)$ \\
2 & $5(5.9)$ \\
3 & $2(2.4)$ \\
More than 3 & $33(38.8)$ \\
\hline Ascites present & $6(7.1)$ \\
\hline
\end{tabular}

None of the cases had acoustic shadows present.

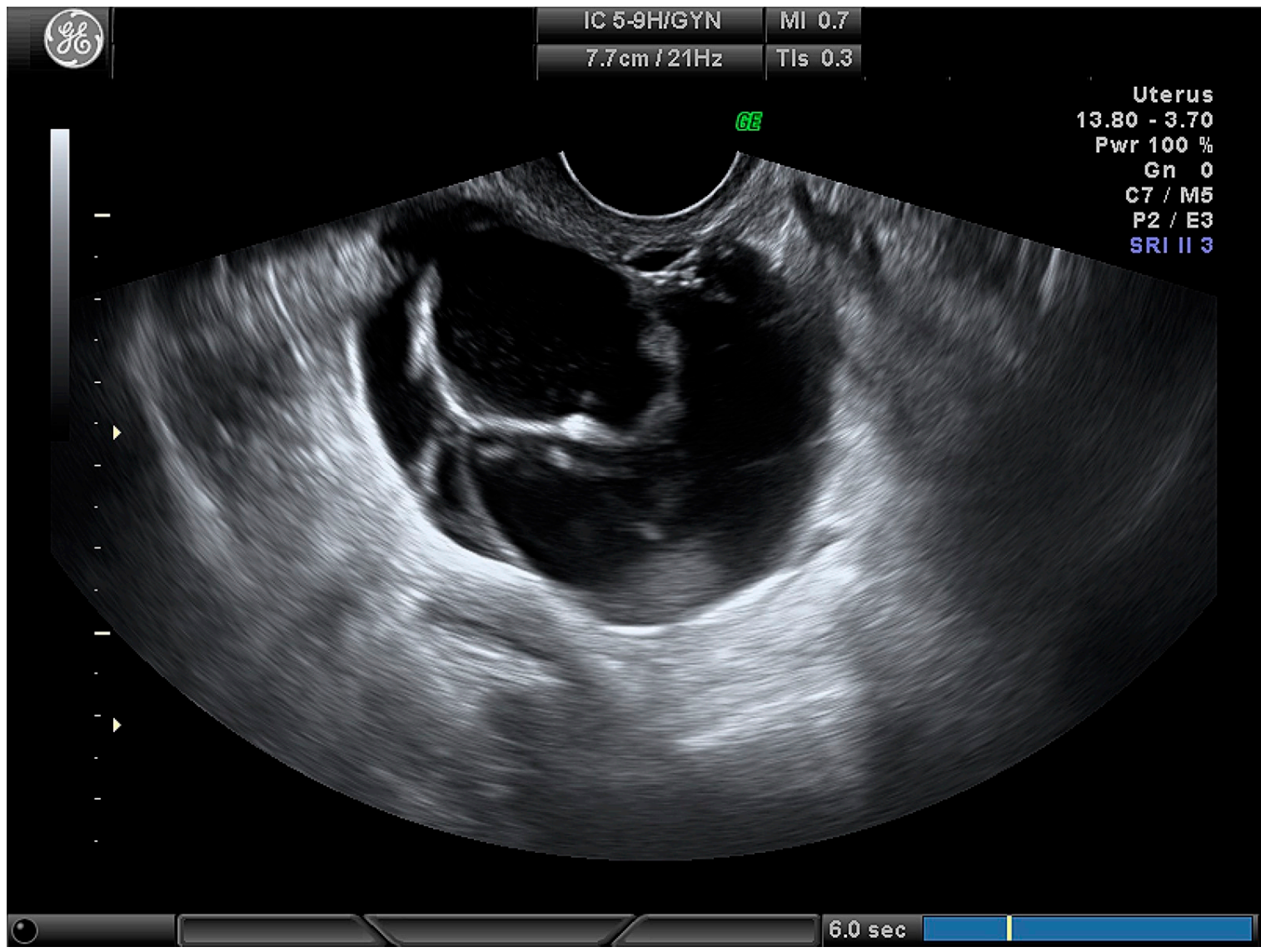

Figure 3. Multilocular cystic ovarian lesion. 


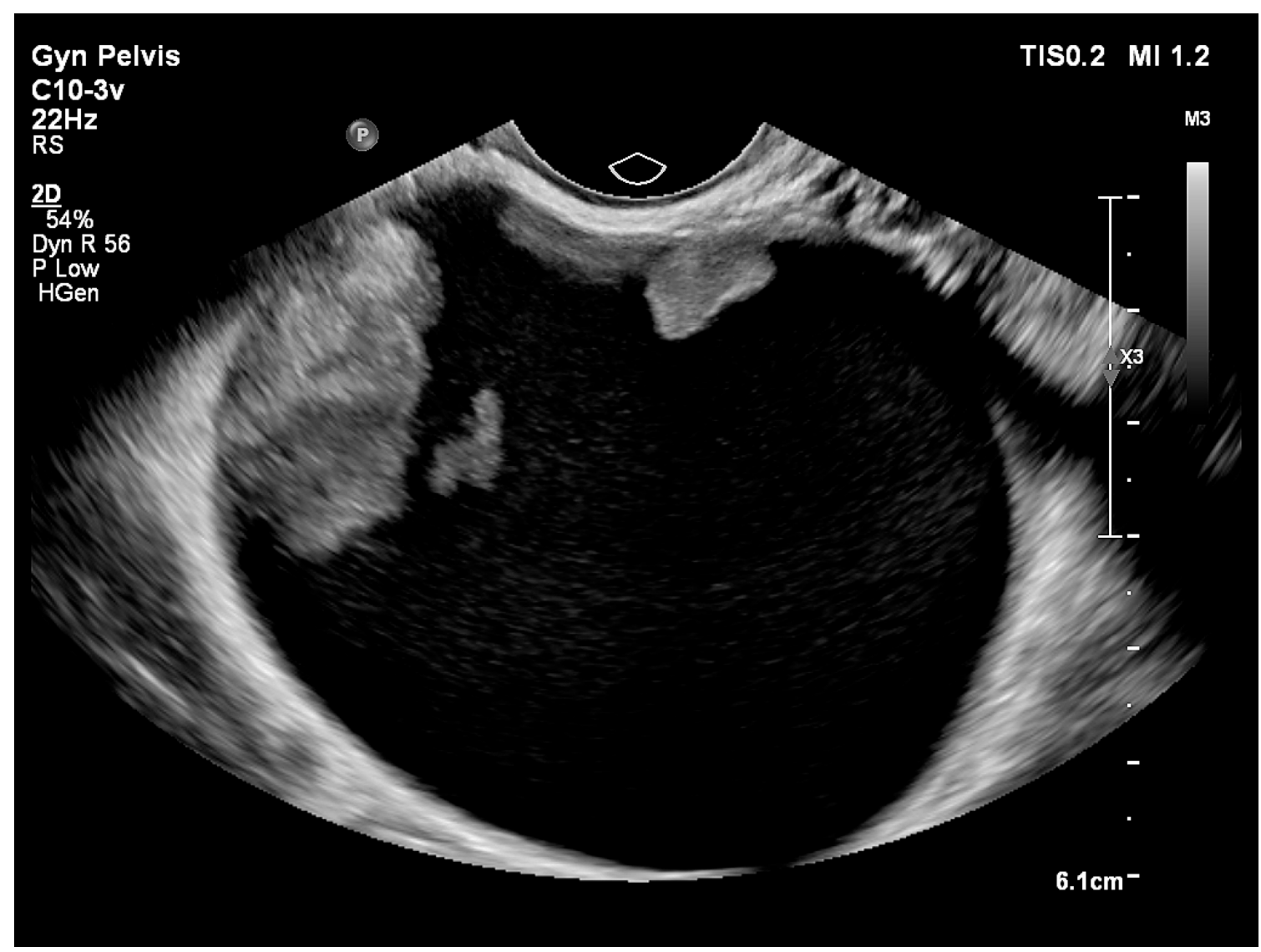

Figure 4. Unilocular cystic-solid ovarian lesion.

Table 3. Ultrasound features of the different BOT histological subtypes.

\begin{tabular}{|c|c|c|c|}
\hline \multirow{3}{*}{ Ultrasound Feature } & Serous BOT & Mucinous BOT & \multirow{3}{*}{ Serous vs. Mucinous } \\
\hline & $(n=57)$ & $(n=22)$ & \\
\hline & \multicolumn{2}{|c|}{ Median (Range) or $n(\%)$} & \\
\hline \multicolumn{4}{|l|}{ Type of tumour: } \\
\hline Cystic & $7(12.3)$ & $12(54.5)$ & $p<0.001$ \\
\hline Cystic-solid & $50(87.7)$ & $10(45.5)$ & \\
\hline \multicolumn{4}{|l|}{ Number of locules: } \\
\hline Uniloculcar & $32(56.1)$ & $5(22.7)$ & $p=0.008$ \\
\hline Multilocular & $25(43.9)$ & $17(77.3)$ & \\
\hline More than 10 locules present & $14(24.6)$ & $14(63.6)$ & $p=0.001$ \\
\hline Presence of papillary projections & $46(80.7)$ & $8(36.4)$ & $p<0.001$ \\
\hline Maximal diameter of the tumour (mm) & $\begin{array}{c}72.0 \\
(20.5-639.0)\end{array}$ & $135(25.7-300)$ & $p=0.003$ \\
\hline Maximal diameter of solid part (mm) & $15.0(3.0-73.0)$ & $21.5(4.0-66.0)$ & $p=0.003$ \\
\hline
\end{tabular}

\subsection{Performance of ADNEX Model According to Absolute and Relative Risk}

The results of ADNEX model-classified cases are presented in Table 4.

The performance of ADNEX model based on absolute risk (AR) depends on the selected cut-off value for the malignancy risk. More encompassing cut-off values allow the model to differentiate BOTs better with an additional number of correctly classified cases, compared to a stricter cut-off value. The results of the ADNEX model performance with the selected different cut-off values are shown in Table 5. 
Table 4. Performance of the ADNEX (Assessment of Different NEoplasias in the adneXa) model according to relative risk (RR) and absolute risk (AR) with different cut-offs.

\begin{tabular}{cccccc}
\hline \multirow{2}{*}{ ADNEX Result } & \multicolumn{3}{c}{ Number (\%) of Cases According to AR with } & \multirow{2}{*}{$\begin{array}{c}\text { Number (\%) of Cases } \\
\text { Different Cut-Off Values }\end{array}$} & \begin{tabular}{c} 
According to RR \\
\cline { 2 - 5 }
\end{tabular} \\
\cline { 2 - 5 } & $\mathbf{3 \%}$ & $\mathbf{5 \%}$ & $\mathbf{1 0 \%}$ & $\mathbf{2 0 \%}$ & $13(16.7 \%)$ \\
\hline Benign & $1(1.3 \%)$ & $5(6.4 \%)$ & $12(15.4 \%)$ & $21(26.9 \%)$ & $59(75.6 \%)$ \\
\hline BOT & $67(85.9 \%)$ & $63(80.8 \%)$ & $56(71.2 \%)$ & $47(60.3 \%)$ & $5(6.4 \%)$ \\
\hline Stage I invasive Ca & $3(3.8 \%)$ & $3(3.8 \%)$ & $3(3.8 \%)$ & $3(3.8 \%)$ & $1(1.3 \%)$ \\
\hline $\begin{array}{c}\text { Stage II-IV } \\
\text { invasive Ca }\end{array}$ & $7(9.0 \%)$ & $7(9.0 \%)$ & $7(9.0 \%)$ & $7(9.0 \%)$ & \\
\hline
\end{tabular}

Table 5. Changes of ADNEX model performance by AR with different cut-offs.

\begin{tabular}{cccc}
\hline Cut-Off & $\begin{array}{c}\text { Correctly Classified } \\
\text { BOT Cases } N \text { (\%) }\end{array}$ & $\begin{array}{c}\text { Absolute Change in } \\
\text { Case Number (\%) }\end{array}$ & $p$-Value \\
\hline $20 \%$ & $47(60.3 \%)$ & - & - \\
\hline $10 \%$ & $56(71.2 \%)$ & $+9(11.5 \%)$ & 0.004 \\
\hline $5 \%$ & $63(80.8 \%)$ & $+7(8.97 \%)$ & 0.016 \\
\hline $3 \%$ & $67(85.9 \%)$ & $+4(5.1 \%)$ & 0.375 \\
\hline
\end{tabular}

When relative risk (RR) was used to classify the tumours, 59 (75.6\%) cases were identified correctly. The comparison of ADNEX performance using RR as a reference and AR with different cut-off values is presented in Table 6 .

Table 6. Comparison of ADNEX performance between RR as a reference * and AR with different cut-off values.

\begin{tabular}{ccc}
\hline Cut-Off Value & $\begin{array}{c}\text { Difference of the Case Number } \\
\text { According to AR }\end{array}$ & $p$-Value \\
\hline $20 \%$ & $-12(15.4 \%)$ & $<0.001$ \\
\hline $10 \%$ & $-3(3.9 \%)$ & 0.375 \\
\hline $5 \%$ & $+4(5.1 \%)$ & 0.388 \\
\hline $3 \%$ & $+7(9.0 \%)$ & 0.118 \\
\hline$*$ reference RR result-59 $(75.6 \%)$ correctly classified BOT cases.
\end{tabular}

\subsection{The Value of Ca125 Marker in ADNEX Model for BOT Classification}

According to AR with selected cut-off value of $10 \%$, without Ca125 added, the ADNEX model correctly classified $69.4 \%$ of the cases. With Ca125 added to the model, the number of correctly classified cases increased to 71.8\%; however, the difference was insignificant $(p=0.629)$.

According to RR, the model without Ca125 classified 72.9\% of the BOT cases correctly. An addition of Ca125 increased the performance of the model to $75.6 \%$. However, this change was also insignificant $(p=0.453)$.

\subsection{The Performance of the ADNEX Model between Different Histological Subtypes of BOTs}

The results of the performance of the ADNEX model for different histological types of BOTs are presented in Table 7.

Ca125 insignificantly increased the number of correctly classified BOT histological subtypes. According to AR, the appliance of Ca125 resulted in additional $7.02 \%$ of serous and of $3.1 \%$ of mucinous 
BOTs $(p=0.454)$; according to RR, applying Ca125 resulted in an additional $8.8 \%$ of serous and of $9.1 \%$ of mucinous BOTs $(p=0.453)$.

Table 7. ADNEX model performance in serous and mucinous BOT groups.

\begin{tabular}{cccc}
\hline Without Ca125 & $\begin{array}{c}\text { Serous BOTs } \\
(\boldsymbol{n}=\mathbf{5 7})\end{array}$ & $\begin{array}{c}\text { Mucinous BOTs } \\
(\boldsymbol{n}=\mathbf{2 2})\end{array}$ & $\begin{array}{c}\boldsymbol{p} \text {-Value } \\
\text { (Serous vs. Mucinous) }\end{array}$ \\
\hline AR (cut-off 10\%) & $40(70.2 \%)$ & $16(72.7 \%)$ & 0.823 \\
\hline RR & $44(77.2 \%)$ & $15(68.2 \%)$ & 0.409 \\
\hline$p$-value (AR vs. RR) & 0.289 & 1.000 & - \\
\hline With Ca125 & $\begin{array}{c}\text { Serous BOTs } \\
(\boldsymbol{n}=53)\end{array}$ & $\begin{array}{c}\text { Mucinous BOTs } \\
(\boldsymbol{n}=\mathbf{2 0})\end{array}$ & $\begin{array}{c}\boldsymbol{p} \text {-Value } \\
\text { (Serous vs. Mucinous) }\end{array}$ \\
\hline AR (cut-off 10\%) & $40(75.5 \%)$ & $15(75.0 \%)$ & 0.967 \\
\hline RR & $42(79.2 \%)$ & $15(75.0 \%)$ & 0.696 \\
\hline$p$-value (AR vs. RR) & 0.625 & 1.000 & - \\
\hline
\end{tabular}

\section{Discussion}

The results of our study have demonstrated a good overall performance of the ADNEX model for discriminating BOTs from other ovarian masses. To our knowledge, it is the only study on the performance of the ADNEX model with a representable set of BOT cases carried in an external center.

A more encompassing cut-off for malignancy values, used to differentiate BOTs according to AR, resulted in lower number of BOT cases wrongly classified as benign tumours. However, the difference was only significant between $10 \%$ and $20 \%$ cut-off values. It was also noted that the number of wrongly classified tumours in malignant categories (stage I and stage II-IV invasive carcinomas) remained the same with all different cut-off values used.

The performance of the ADNEX model based on RR was significantly better compared to AR with a cut-off value of $20 \%$. The better performance of RR was also noted when AR with a $10 \%$ cut-off value was used; however, this was not significant. On the other hand, AR with 5\% and 3\% cut-offs performed better than RR, although these differences were insignificant. It was previously noted that due to the low overall prevalence of some types of ovarian malignancies, such as metastatic lesions, as well as BOTs, RR can be a more informative tool [11]. In our study, we noticed the advantages of RR compared to AR only in the groups with lower cut-off values. Nevertheless, prospective evaluation and bigger sample studies are needed to address this issue.

The value of Ca125 in the diagnosis of BOTs is still controversial. While most of the studies report slightly elevated Ca125 levels, especially in serous BOT groups [2], Eltabbakh et al. and more recent publication by Morroti et al. emphasize that in cases of early invasive cancer and BOTs, the levels of Ca125 marker overlap [3,12]. However, it has been noted that Ca125 could be an independent prognostic factor for peritoneal implants $[13,14]$. In the ADNEX model, the Ca125 is an optional feature, but the authors state that missing the value of this marker will decrease the accuracy of the model to discriminate between stage II-IV invasive ovarian tumours and other malignancy types [10]. Our results showed the tendency of Ca125 to increase the number of correctly classified BOTs when both AR and RR were employed. It has been also demonstrated with different histological subtypes of BOTs; however, none of these results were statistically significant.

Concerning the ADNEX model's performance in different subtypes of BOTs, this is the first study addressing the issue. The greyscale ultrasound features of both subtypes in our study agrees with those noted by the other authors: serous BOTs were mainly unilocular, solid tumours with papillary projections $[9,15]$; while mucinous tumours were described as multilocular cysts, significantly larger than serous tumours [16]. Regardless of the different sonographic features of these subgroups, our results showed a similar performance of the ADNEX model for both histological subtypes, with no significant differences. 
Obviously, the retrospective nature of this study is a major limitation; however, we assume that, reviewing the recorded data and protocols of ultrasound examinations adherent to IOTA terminology and measurement technique, we have retrieved credible ultrasound characteristics of adnexal masses.

\section{Conclusions}

The IOTA ADNEX model performs well in discriminating BOTs from other ovarian tumours. The calculation based on RR or AR with the cut-off value of at least $10 \%$ should be used when evaluating for BOTs. Ca125 has a tendency to improve the results. The ADNEX model performs equally well in both serous and mucinous BOTs.

Author Contributions: Conceptualization, A.G. and M.G.; methodology A.G., M.G., D.V., and J.R.N.; data curation M.G., D.G., A.C., and V.A.; writing-original draft preparation A.G. and M.G.; writing-review and editing, S.P., T.B., D.V., and R.J.N.; visualization, A.G. and M.G.; supervision R.J.N. All authors have read and agreed to the published version of the manuscript.

Funding: This research received no external funding.

Acknowledgments: The authors are grateful to those who helped with the writing of this manuscript. Thanks to all the peer reviewers and editors for their opinions and suggestions.

Conflicts of Interest: The authors declare no conflict of interest.

\section{References}

1. Tinelli, R.; Tinelli, A.; Tinelli, F.G.; Cicinelli, E.; Malvasi, A. Conservative surgery for borderline ovarian tumors: A review. Gynecol. Oncol. 2006, 100, 185-191. [CrossRef] [PubMed]

2. Trimble, C.L.; Trimble, E.L. Management of epithelial ovarian tumors of low malignant potential. Gynecol. Oncol. 1994, 55, S52-S61. [CrossRef] [PubMed]

3. Morotti, M.; Menada, M.V.; Gillott, D.J.; Venturini, P.L.; Ferrero, S. The preoperative diagnosis of borderline ovarian tumors: A review of current literature. Arch. Gynecol. Obstet. 2012, 285, 1103-1112. [CrossRef] [PubMed]

4. Skirnisdottir, I.; Garmo, H.; Wilander, E.; Holmberg, L. Borderline ovarian tumors in Sweden 1960-2005: Trends in incidence and age at diagnosis compared to ovarian cancer. Int. J. Cancer 2008, 123, 1897-1901. [CrossRef] [PubMed]

5. Colombo, N.; Sessa, C.; du Bois, A.; Ledermann, J.; McCluggage, W.G.; McNeish, I.; Morice, P.; Pignata, S.; Ray-Coquard, I.; Vergote, I.; et al. ESMO-ESGO consensus conference recommendations on ovarian cancer: Pathology and molecular biology, early and advanced stages, borderline tumours and recurrent diseaset. Ann. Oncol. 2019, 30, 672-705. [CrossRef] [PubMed]

6. Vasconcelos, I.; de Sousa Mendes, M. Conservative surgery in ovarian borderline tumours: A meta-analysis with emphasis on recurrence risk. Eur. J. Cancer 2015, 51, 620-631. [CrossRef] [PubMed]

7. Kaijser, J. Towards an evidence-based approach for diagnosis and management of adnexal masses: Findings of the International Ovarian Tumour Analysis (IOTA) studies. Facts Views Vis. Obgyn 2015, 7, 42-59. [PubMed]

8. Timmerman, D.; Schwärzler, P.; Collins, W.P.; Claerhout, F.; Coenen, M.; Amant, F.; Vergote, I.; Bourne, T.H. Subjective assessment of adnexal masses with the use of ultrasonography: An analysis of interobserver variability and experience. Ultrasound Obstet. Gynecol. 1999, 13, 11-16. [CrossRef] [PubMed]

9. Fruscella, E.; Testa, A.C.; Ferrandina, G.; De Smet, F.; Van Holsbeke, C.; Scambia, G.; Zannoni, G.F.; Ludovisi, M.; Achten, R.; Amant, F.; et al. Ultrasound features of different histopathological subtypes of borderline ovarian tumors. Ultrasound Obstet. Gynecol. 2005, 26, 644-650. [CrossRef] [PubMed]

10. Van Calster, B.; Van Hoorde, K.; Valentin, L.; Testa, A.C.; Fischerova, D.; Van Holsbeke, C.; Savelli, L.; Franchi, D.; Epstein, E.; Kaijser, J.; et al. Evaluating the risk of ovarian cancer before surgery using the ADNEX model to differentiate between benign, borderline, early and advanced stage invasive, and secondary metastatic tumours: Prospective multicentre diagnostic study. BMJ 2014, 349, 5920. [CrossRef] [PubMed]

11. Van Calster, B.; Van Hoorde, K.; Froyman, W.; Kaijser, J.; Wynants, L.; Landolfo, C.; Anthoulakis, C.; Vergote, I.; Bourne, T.; Timmerman, D.; et al. Practical guidance for applying the ADNEX model from the IOTA group to discriminate between different subtypes of adnexal tumors. Facts Views Vis. Obgyn 2015, 7, 32-41. [PubMed] 
12. Eltabbakh, G.H.; Yadav, P.R.; Morgan, A. Clinical picture of women with early stage ovarian cancer. Gynecol. Oncol. 1999, 75, 476-479. [CrossRef]

13. Fotopoulou, C.; Sehouli, J.; Ewald-Riegler, N.; de Gregorio, N.; Reuss, A.; Richter, R.; Mahner, S.; Kommoss, F.; Schmalfeldt, B.; Fehm, T.; et al. The Value of Serum CA125 in the Diagnosis of Borderline Tumors of the Ovary: A Subanalysis of the Prospective Multicenter ROBOT Study. Int. J. Gynecol. Cancer 2015, 25, 1248-1252. [CrossRef]

14. May, J.; Skorupskaite, K.; Congiu, M.; Ghaoui, N.; Walker, G.A.; Fegan, S.; Martin, C.W.; O'Donnell, R.L. Borderline Ovarian Tumors: Fifteen Years' Experience at a Scottish Tertiary Cancer Center. Int. J. Gynecol. Cancer 2018, 28, 1683-1691. [CrossRef] [PubMed]

15. Moro, F.; Baima Poma, C.; Zannoni, G.F.; Vidal Urbinati, A.; Pasciuto, T.; Ludovisi, M.; Moruzzi, M.C.; Carinelli, S.; Franchi, D.; Scambia, G.; et al. Imaging in gynecological disease (12): Clinical and ultrasound features of invasive and non-invasive malignant serous ovarian tumors. Ultrasound Obstet. Gynecol. 2017, 50, 788-799. [CrossRef]

16. Pascual, A.; Guerriero, S.; Rams, N.; Juez, L.; Ajossa, S.; Graupera, B.; Hereter, L.; Cappai, A.; Pero, M.; Perniciano, M.; et al. Clinical and ultrasound features of benign, borderline, and malignant invasive mucinous ovarian tumors. Eur. J. Gynaecol. Oncol. 2017, 38, 382-386.

Publisher's Note: MDPI stays neutral with regard to jurisdictional claims in published maps and institutional affiliations.

(C) 2020 by the authors. Licensee MDPI, Basel, Switzerland. This article is an open access article distributed under the terms and conditions of the Creative Commons Attribution (CC BY) license (http://creativecommons.org/licenses/by/4.0/). 